\title{
Systematic Uncertainties in the Analysis of the Reactor Neutrino Anomaly
}

\author{
A.C. Hayes ${ }^{1}$, J.L. Friar ${ }^{1}$, G.T. Garvey ${ }^{1}$, Gerard Jungman ${ }^{1}$, G. Jonkmans ${ }^{2}$ \\ ${ }^{1}$ Los Alamos National Laboratory, Los Alamos, NM, USA 87545 \\ ${ }^{2}$ AECL, Chalk River Laboratories, Chalk River, Ontario, Canada, K0J 1 J0
}

\begin{abstract}
We examine uncertainties in the analysis of the reactor neutrino anomaly, wherein it is suggested that only about $94 \%$ of the emitted antineutrino flux was detected in short baseline experiments. We find that the form of the corrections that lead to the anomaly are very uncertain for the $30 \%$ of the flux that arises from forbidden decays. This uncertainty was estimated in four ways, is larger than the size of the anomaly, and is unlikely to be reduced without accurate direct measurements of the antineutrino flux. Given the present lack of detailed knowledge of the structure of the forbidden transitions, it is not possible to convert the measured aggregate fission beta spectra to antineutrino spectra to the accuracy needed to infer an anomaly. Neutrino physics conclusions based on the original anomaly need to be revisited, as do oscillation analyses that assumed that the antineutrino flux is known to better than approximately $4 \%$.
\end{abstract}

The term "reactor neutrino anomaly" first appeared in a publication by G. Mention et al. [1], where it generally referred to the $3 \sigma$ deficit of neutrinos detected in short-baseline reactor neutrino experiments relative to the number predicted. The predicted number of detected neutrinos has evolved upward over time, largely as a consequence of a predicted increase in the energy of the neutrino flux and an increased $\bar{\nu}_{e}+p \rightarrow n+e^{+}$cross section associated with smaller values for the neutron lifetime. This cross section is used to infer the neutrino flux in a presumably well-characterized detector. The changes in the predicted neutrino flux are mostly associated with improved knowledge of the beta decays of the isotopes created in fission reactors. Such an anomaly would potentially be extremely significant, if a shortfall in the detected neutrino flux could be ascribed to $\bar{\nu}_{e}$ oscillation into a light sterile neutrino with a mass of about $1 \mathrm{eV}$.

There is an extensive recent literature dealing with the reactor anomaly, starting with a seminal paper by Mueller et al. 22 that reexamined the reactor antineutrino flux. The latter publication sought to improve the earlier flux estimates based on the ILL on-line measurements [3 5] of the integral beta spectrum of the fission products. An antineutrino spectrum can be inferred from a beta spectrum provided one knows the linear combination of operators involved in the decay, the end-point energy, and the nuclear charge. The fission beta spectra involve about 6000 beta transitions, of which about 1500 are forbidden [6]. Clearly some assumptions are required in order to infer the fission antineutrino flux. The improvements [1, 2] on the earlier analyses of ILL integral measurements led to an increased energy of the antineutrino flux, which was subsequently verified in an independent analysis [7].

The present contribution examines the consequences of the forbidden transitions known to be present (at the $30 \%$ level) in the beta decay of fission products. We analyze the antineutrino flux, using a first-principles derivation of the finite size (FS) and weak magnetism (WM) corrections that were the main focus of the analyses in
Refs. 2, 7. In addition, we examine the shape factors needed to describe the forbidden transitions. We find that the forbidden transitions introduce a large uncertainty in the predicted antineutrino flux irrespective of whether the antineutrino spectra were deduced using nuclear databases or by inverting measured aggregate fission beta spectra. As detailed below, this finding results from the fact that the corrections are nuclear-operator dependent and that an undetermined combination of matrix elements contributes to non-unique forbidden transitions.

The beta-decay spectrum $S$ for a single transition in nucleus $(Z, A)$ with end-point energy $E_{0}=E_{e}+E_{\nu}$ is

$S\left(E_{e}, Z, A\right)=S_{0}\left(E_{e}\right) F\left(E_{e}, Z, A\right) C\left(E_{e}\right)\left(1+\delta\left(E_{e}, Z, A\right)\right)$,

where $S_{0}=G_{F}^{2} p_{e} E_{e}\left(E_{0}-E_{e}\right)^{2} / 2 \pi^{3}, E_{e}\left(p_{e}\right)$ is the electron total energy (momentum), $F\left(E_{e}, Z, A\right)$ is the Fermi function needed to account for the Coulomb interaction of the outgoing electron with the charge of the daughter nucleus, and $C\left(E_{e}\right)$ is a shape factor 8 for forbidden transitions due to additional lepton momentum terms. For allowed transitions $C(E)=1$. The term $\delta\left(E_{e}, Z, A\right)$ represents fractional corrections to the spectrum that were the central focus of the original anomaly studies. The primary corrections to beta decay are radiative, finite size, and weak magnetism, or $\delta\left(E_{e}, Z, A\right)=$ $\delta_{\mathrm{rad}}+\delta_{\mathrm{FS}}+\delta_{\mathrm{WM}}$.

Before discussing the details of the corrections $C\left(E_{e}\right)$ and $\delta\left(E_{e}\right)$, we briefly summarize the treatments used in earlier work. The radiative corrections as derived by Sirlin 9 were included in the description of the beta spectra (though not in the antineutrino spectra) in the original analyses of Schreckenbach et al. 3 - 5. In the later ILL work [4, 5] an approximation for the FS and WM corrections was included by first deducing the antineutrino spectrum from the measured beta spectra without these corrections, and then applying a linear correction to the deduced antineutrino spectrum of the form, $\delta_{\mathrm{FS}}+\delta_{\mathrm{WM}}=0.0065\left(E_{\nu}-4 \mathrm{MeV}\right)$. In that work no cor- 
rections were made for the shape factors $C\left(E_{e}\right)$. In the analyses of Refs. 2, 7, 10] an approximation (derived by Vogel [11]) for the FS and WM corrections was applied on a transition-by-transition basis. In Refs. 2, 10. the shape factor appropriate for unique forbidden transitions was used for all forbidden transitions. In Ref. [7] it was argued that these shape factors only play a small role in inferring antineutrino spectra from measured beta spectra because for vanishing electron mass, $m_{e}$, they are symmetric under $E_{e} \leftrightarrow E_{\nu}$. In the present work, we derived $a b$ initio analytic expressions for the FS and WM corrections for allowed GT transitions, as well as WM and shape factors for first-forbidden GT operators. We used the radiative corrections derived by Sirlin [9].

We now turn to the form of the corrections. The attractive Coulomb interaction increases the electron density near the nuclear surface and increases the beta-decay rate, while the finite nuclear size decreases the electron density and decreases the rate (relative to the pointnucleus Fermi function). Using first-order perturbation theory in $Z \alpha$, we find that the finite-size correction to the Fermi function, $\delta_{\mathrm{FS}}$, for allowed GT transitions is

$$
\delta_{\mathrm{FS}}=-\frac{3}{2} \frac{Z \alpha}{\hbar c}\langle r\rangle_{(2)}\left(E_{e}-\frac{E_{\nu}}{27}+\frac{m_{e}^{2} c^{4}}{3 E_{e}}\right) .
$$

The quantity $\langle r\rangle_{(2)}=\int d^{3} r \rho_{W}(r) \int d^{3} s \rho_{\mathrm{ch}}(s)|\vec{r}-\vec{s}|$ is the first moment of the convoluted nuclear weak and charge densities (called a Zemach moment [12]). We assume uniform distributions of radius $R$ for the weak and charge densities, for which $\langle r\rangle_{(2)}=\frac{36}{35} R[13$. The FS corrections do depend on the beta-decay operator, but in this work we always use Eq. (2) and $R=1.2 A^{1 / 3} \mathrm{fm}$.

The WM correction arises from the interference of the magnetic moment distribution of the vector current, $\vec{J}_{V}=\vec{\nabla} \times \vec{\mu}$, with the spin distribution $\vec{\Sigma}$ of the axial current. We derived the WM corrections for allowed and first-forbidden operators. There are four possible operators in the case of first-forbidden GT transitions, and all have well-defined WM corrections, as listed in Table 1. Our FS and WM corrections for allowed GT transitions are identical to those derived by Holstein [14, and differ from the forms used in $2,17,10,11$. The first-forbidden shape factors, $C\left(E_{e}\right)$, which depend on the operator in question, were derived and are displayed in Table 1. To determine the implications of the corrections we take advantage of the fact that the ENDF/B-VII.1 decay library [6] contains branching ratios and end-point energies for over $90 \%$ of the transitions making up the full spectra. Thus, the library allows a representative estimate of the effect of the corrections, provided we know what assumptions to make about the forbidden transitions.

We first fit the Schreckenbach 4 ] electron spectrum for ${ }^{235} \mathrm{U}$ before examining the effect of the corrections in an analysis based on the summation of the known beta branches of the fission products. We include the corrections both with and without a treatment of the for- bidden transitions. We fit the beta spectrum with 40 fictitious equally spaced end-point energies using nonnegative least-squares fitting [15]. The charge $\mathrm{Z}$ associated with each end-point is assigned according to the method given in [4. There is no unique physical prescription for beta-decay operator assignments to the fictitious end-points. For this reason we examine four prescriptions: (1) all transitions are assumed to be allowed; (2) all end-point energies can be associated with either an allowed or forbidden transition; (3) 30\% of the branches are selected to be forbidden at equal energy intervals; (4) $30 \%$ of the branches are selected to be forbidden with a bias towards higher energies. In addition, we examine fits in which the operator determining the forbidden decays was taken to be $[\Sigma, r]^{0-},[\Sigma, r]^{1-},[\Sigma, r]^{2-}$ or a combination of these. As a natural consequence of the non-negative least-squares procedure, the fit results in a significant fraction of the end-points having zero amplitude. The subset of end-points with non-zero amplitudes varies depending on the operator assignment.

We find excellent fits to the electron spectrum in all cases. However, different treatments of the forbidden transitions can lead to antineutrino spectra that differ both in shape and magnitude at about the $4 \%$ level. Two examples are shown in Fig.1, where we present the fits obtained when the WM and FS corrections are included. In one case all transitions are assumed to be allowed, while in the second case the best fit results from about $25 \%$ forbidden decays. For the assumption of all allowed transitions, we see a systematic increase of about $2.2 \%$ in the number of antineutrinos relative to Schreckenbach, while including forbidden transitions leads to no increase relative to Schreckenbach. Other prescriptions for the forbidden transitions lead to changes relative to the Schreckenbach antineutrino spectrum ranging from $0-4 \%$, including changes in the shape of the antineutrino spectrum. These examples help to clarify the difficulty in inferring with high certainty the antineutrino spectrum from a measured electron spectrum when information on the forbidden transitions is not available.

In calculating the aggregate fission spectra from the database library an analogous uncertainty arises because detailed structure information does not exist for the majority of the roughly 1500 forbidden transitions. In addition, several of the transitions are forbidden at second or higher order, for which analytic corrections are not available. Thus, there is no clear prescription for applying the corrections to this component of the spectra, and it is more beneficial to examine the effect of different approximations in order to estimate the uncertainty involved. In all approximations we treat unique forbidden transitions as unique first-forbidden GT transitions, and treat nonunique forbidden transitions in one of the following ways: (1) as allowed GT; (2) as unique first-forbidden GT with the operator $[\Sigma, r]^{2-} ;(3)$ with the operator $[\Sigma, r]^{0-} ;(4)$ 
TABLE I: The shape factors and leading-order weak magnetism corrections to allowed and first-forbidden Gamow-Teller beta decays are shown in the top panel. The shape factors for allowed and first-forbidden Fermi beta decays are shown in the bottom panel. All agree with Ref. [16] for $Z=0$. The entries for $\vec{J}_{V}$ and $\rho_{A}$ are discussed in [17]. The weak magnetism correction for $\vec{J}_{V}$ involves the unknown overlap of very different $1^{-}$matrix elements and is therefore not listed. The nucleon isovector magnetic moment is $\mu_{v}=4.7, M_{N}$ is the nucleon mass, $g_{A}$ is the axial vector coupling constant, and $\beta=p_{e} / E_{e}$. No meson currents were used in the magnetic moment operator, and a truncated orbital current led to the factor of " $1 / 2$ " in $\delta_{\mathrm{WM}}$.

\begin{tabular}{ccccc} 
Classification & $\Delta J^{\pi}$ & Operator & Shape Factor $C\left(E_{e}\right)$ & Fractional Weak Magnetism Correction $\delta_{\mathrm{WM}}\left(E_{e}\right)$ \\
\hline \hline Allowed GT & $1^{+}$ & $\Sigma \equiv \sigma \tau$ & 1 & $\frac{2}{3}\left[\frac{\mu_{v}-1 / 2}{M_{N} g_{A}}\right]\left(E_{e} \beta^{2}-E_{\nu}\right)$ \\
Non-unique $1^{\text {st }}$ Forbidden GT & $0^{-}$ & {$[\Sigma, r]^{0-}$} & $p_{e}^{2}+E_{\nu}^{2}+2 \beta^{2} E_{\nu} E_{e}$ & 0 \\
Non-unique $1^{s t}$ Forbidden $\rho_{A}$ & $0^{-}$ & {$[\Sigma, r]^{0-}$} & $\lambda E_{0}^{2}$ & 0 \\
Non-unique $1^{\text {st }}$ Forbidden GT & $1^{-}$ & {$[\Sigma, r]^{1-}$} & $p_{e}^{2}+E_{\nu}^{2}-\frac{4}{3} \beta^{2} E_{\nu} E_{e}$ & {$\left[\frac{\mu_{v}-1 / 2}{M_{N} g_{A}}\right]\left[\frac{\left(p_{e}^{2}+E_{\nu}^{2}\right)\left(\beta^{2} E_{e}-E \nu\right)+2 \beta^{2} E_{e} E_{\nu}\left(E_{\nu}-E_{e}\right) / 3}{\left(p_{e}^{2}+E_{\nu}^{2}-4 \beta^{2} E_{\nu} E_{e} / 3\right)}\right.$} \\
Unique $1^{s t}$ Forbidden GT & $2^{-}$ & {$[\Sigma, r]^{2-}$} & $p_{e}^{2}+E_{\nu}^{2}$ & $\frac{3}{5}\left[\frac{\mu_{v}-1 / 2}{M_{N} g_{A}}\right]\left[\frac{\left(p_{e}^{2}+E_{\nu}^{2}\right)\left(\beta^{2} E_{e}-E_{\nu}\right)+2 \beta^{2} E_{e} E_{\nu}\left(E_{\nu}-E_{e}\right) / 3}{\left(p_{e}^{2}+E_{\nu}^{2}\right)}\right.$ \\
\hline Allowed F & $0^{+}$ & $\tau$ & 1 & 0 \\
Non-unique $1^{s t}$ Forbidden F & $1^{-}$ & $r \tau$ & $p_{e}^{2}+E_{\nu}^{2}+\frac{2}{3} \beta^{2} E_{\nu} E_{e}$ & 0 \\
Non-unique $1^{s t}$ Forbidden $\vec{J}_{V}$ & $1^{-}$ & $r \tau$ & $E_{0}^{2}$ & - \\
\hline
\end{tabular}
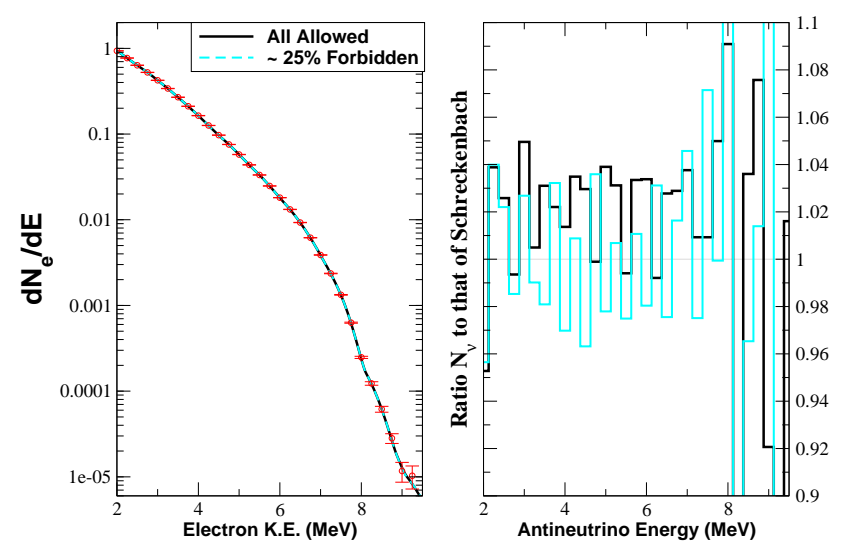

FIG. 1: The fit to the electron spectrum for ${ }^{235} \mathrm{U}$ (left) for two different assumptions on how to treat forbidden transitions, and the ratio of the corresponding antineutrino spectra to that of Schreckenbach (right). The electron data are those of 4]. The electron spectra are fit assuming (a) all allowed GT branches, or (b) up to $30 \%$ forbidden GT transitions. In both cases the WM and FS corrections are included. When folded over the neutrino detection cross section, the case for all allowed (25\% forbidden) transitions results in a $2.2 \%(0.06 \%)$ increase in the number of detectable antineutrinos.

with the operator $[\Sigma, r]^{1-}$. None of the these treatments is correct, but they provide estimates for changes in the spectra induced by forbidden transitions.

The aggregate fission beta spectrum under equilibrium reactor burning conditions for a given actinide is determined by the beta spectra $S\left(E_{e}, Z_{i}, A_{i}\right)$ of the individual unstable fission fragments weighted by their cumulative fission yields, $Y_{F_{i}}$ [18:

$$
N_{\beta}\left(E_{e}\right)=\sum_{F_{i}} Y_{F_{i}} S\left(E_{e}, Z_{i}, A_{i}\right)
$$

The beta spectrum $S$ for each fragment $\left(Z_{i}, A_{i}\right)$ summed over all decay branches must be normalized to unity: $\int S(E, Z, A) d E=1$. Thus, Eq. (3) is a statement that under equilibrium burning conditions the beta-decay rates are determined by the fission rate [19. If the antineutrino spectrum is inferred from a measured aggregate beta spectrum, Eq. (3) must be replaced by a sum over a set of end-point energies $\left\{E_{0_{i}}\right\}$, weighted by a fitted set of coefficients $\left\{a_{i}\right\}: N_{\beta}=\sum_{i} a_{i} S\left(E_{e}, E_{0_{i}}\right)$.

There is no unique method for determining the uncertainty in the antineutrino spectrum introduced by the forbidden transitions. Another possibility (in addition to the fitting exercise above) is to consider changes in the bi-variant function $k\left(E_{e}, E_{\nu}\right)$, where $k\left(E_{e}, E_{\nu}\right)=$ $N_{\nu}\left(E_{\nu}\right) / N_{\beta}\left(E_{e}\right)$. If $k\left(E_{e}, E_{\nu}\right)$ only changes by some small percentage for some path in the $\left(E_{e}, E_{\nu}\right)$ plane as we change our treatment of forbidden transitions, then there exists a prescription for inferring the antineutrino spectrum to that accuracy. We calculated the function $k\left(E_{e}, E_{\nu}\right)$ for each of our four assumptions (above). We found no path in the $\left(E_{e}, E_{\nu}\right)$ plane that left $k\left(E_{e}, E_{\nu}\right)$ unchanged by as little as $5 \%$ as our assumptions for the forbidden transitions changed. Fig. (2) depicts the result for the path $E_{\nu}=K_{\beta} \equiv E_{e}-m_{e} c^{2}$ [20]. Similar or larger differences were found for all other paths. The very nonsmooth and non-linear shape of $k$ arises from the shape factors $C\left(E_{e}\right)$.

A third prescription for estimating the uncertainties is to examine the rate of change in the antineutrino spectrum relative to the rate of change in the beta spectrum, using the fact that the beta spectrum is fit to amplitudes $a_{i}$ on a fixed grid of end-point energies $E_{0_{i}}$. We calcu- 


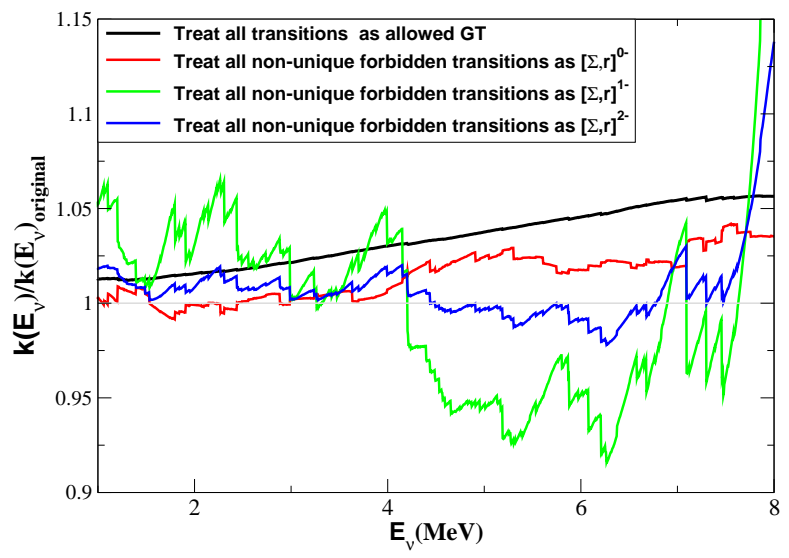

FIG. 2: The ratio of the function $k\left(E_{e}, E_{\nu}\right)$ for ${ }^{235} \mathrm{U}$ using ENDFB/VII.1 relative to using $E_{\nu}=K_{\beta}[20$.

lated $T=\sum_{i}\left[\partial N_{\nu}\left(E_{\nu}\right) / \partial a_{i}\right] /\left[\partial N_{\beta}\left(E_{e}\right) / \partial a_{i}\right]$, and examined the changes in $T$ as the assumptions for the forbidden transitions were varied, and again found no path in $\left(E_{e}, E_{\nu}\right)$ over which the changes were $<5 \%$.

Our final method considers the ratio of the actual antineutrino spectra themselves in Fig. (3), although this method does not take into account the corresponding changes in the beta spectra. We carried out identical analyses of the role of the corrections and the associated uncertainties for the other actinides ${ }^{239,241} \mathrm{Pu}$ and ${ }^{238} \mathrm{U}$, and found very similar results. Fig. (3) shows the change in the total antineutrino spectrum at a representative time [21] in mid-cycle in the reactor burn history. The antineutrino spectra differ significantly, depending on our treatment of the forbidden transitions. The crosssection-weighted spectra are quite distorted, being lower than the Schreckenbach-Vogel 4, 22, 23, spectra up to the peak, and higher or lower above the peak depending on the operator. The actual spectrum is unlikely to be as distorted as in Fig. (3) because no single operator dominates the forbidden transitions.

The original ILL analysis [4] assumed that the $Z$ of the daughter fragments satisfies $Z_{i}=49.5-0.7 E_{0_{i}}-0.09 E_{0_{i}}^{2}$ for $Z_{i} \geq 34$. We find that taking the $Z$ of the fission fragments directly from ENDF/B-VII.1 instead would increase the antineutrino spectrum by less than $1 \%$ for $E_{\nu} \leq 7 \mathrm{MeV}$, and less than $1.5 \%$ for $E_{\nu}>7 \mathrm{MeV}$.

In summary we find that the component of the aggregate fission spectra containing approximately $30 \%$ forbidden transitions introduces a large uncertainty (about $4 \%$ ) in the predicted shape of the antineutrino flux emitted from reactors. We have examined the uncertainties in four different ways. If all forbidden transitions are treated as allowed GT transitions, the antineutrino spectra are systematically increased above $2 \mathrm{MeV}$, as was the
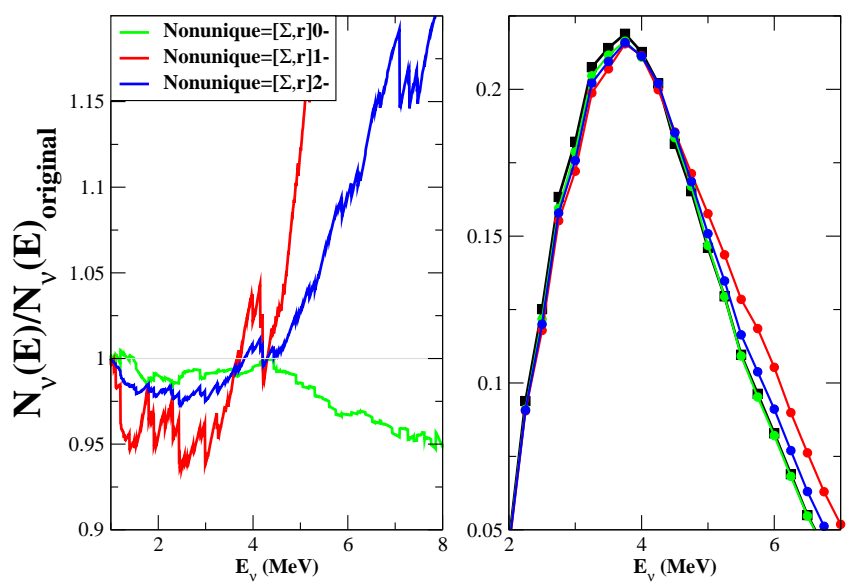

FIG. 3: Different treatments of the forbidden GT transitions contributing to the antineutrino spectrum summed over all actinides in the fission burn in mid-cycle 21 of a typical reactor. The left panel shows the ratio of these antineutrino spectra relative to that using the assumptions of Ref. [4]. The right panel shows the spectra weighted by the detection cross section, where the additional curve in black uses the assumptions of Ref. 4]. The spectra are strongly distorted by the forbidden operators, being lower below the peak and in some cases more than $20 \%$ larger above the peak than Ref. 4]. The corresponding change in the number of detectable antineutrinos relative to [4 is $-0.75 \%, 5.8 \%$ and $1.85 \%$ for the $0^{-}, 1^{-}$, and $2^{-}$forbidden operators, respectively.

conclusion in the earlier papers on the anomaly. However, when the forbidden transitions are treated in various approximations, the shape and magnitude of the spectra are changed significantly. Earlier analyses only looked at one prescription for these transitions. The uncertainty introduced by our lack of knowledge on how to treat these transitions is as large as the size of the anomaly. It should also be noted that there are additional uncertainties due to our oversimplified treatment of the allowed weak magnetism operator (viz., no meson currents and a truncated orbital current), as well as a simplified finite-size treatment for forbidden transitions. These considerations also apply to fission antineutrino fluxes in medium and long baseline reactor experiments, implying a $4 \%$ uncertainty on the fission antineutrino flux in those experiments. Reducing the uncertainty within a purely theoretical framework would be difficult. An improvement will require either direct measurements of the antineutrino flux or a substantial improvement in our knowledge of the dominant forbidden beta transitions. 
[1] G. Mention, et al., Phys. Rev. D 83073006 (2011).

[2] Th. A. Mueller, et al., Phys. Rev. C 83, 054615 (2011).

[3] K. Schreckenbach, H. R. Faust, F. von Feilitzsch, A. A. Hahn, K. Hawerkamp, and J. L. Vuilleumier, Phys. Lett. 99B, 251 (1981); F. von Feilitzsch, A. A. Hahn, and K. Schreckenbach, Phys. Lett. 118B, 162 (1982).

[4] K. Schreckenbach, G. Colvin, W. Gelletly, and F. von Feilitzsch, Phys. Lett. 160B 325 (1985). The data from Table 1 and the antineutrino predictions of this reference will be denoted above simply as "Schreckenbach."

[5] A. A. Hahn, K. Schreckenbach, W. Gelletly, F. von Feilitzsch, G. Colvin, and B. Krusche, Phys. Lett. B218, 365 (1989).

[6] M. B. Chadwick, Nucl. Data Sheets 112, 2887 (2011).

[7] P. Huber, Phys. Rev. C 84, 024617 (2011).

[8] H. F. Schopper, Weak Interactions and Nuclear Beta Decay, North-Holland, Amsterdam, 1966.

[9] A. Sirlin, Phys. Rev. 164, 1767 (1967); A. Sirlin, Phys. Rev. D 84, 014021 (2011).

[10] M. Fallot, S. Cormon, M. Estienne, A. Algora, V. M. Bui, et al., Phys. Rev. Lett. 109, 202504 (2012).

[11] P. Vogel, Phys. Rev. D 29, 1918 (1984) and private communication.

[12] C. Zemach, Phys. Rev. 104, 1771 (1956).

[13] J. L. Friar, Ann. Phys. (N.Y.) 122, 151 (1979).

[14] B. R. Holstein, Phys. Rev. C 9, 1742 (1974); J. L. Friar and I. Sick, Phys. Lett. B579, 285 (2004). Equation (2) of the latter allows conversion between the two results.

[15] C. Lawson and R. Hanson, Solving Least Squares Problems, Prentice-Hall, Englewood Cliffs, New Jersey, 1974.

[16] D. J. Millener, D. E. Alburger, E. K. Warburton, and D. H. Wilkinson, Phys. Rev C 26, 1167 (1982).

[17] Nuclear operators $\vec{J}_{V}$ and $\rho_{A}$ are proportional to a nucleon velocity $\left(p / M_{N}\right)$, have negative parity, and are therefore first-forbidden. Replacing them by the analogous operators proportional to $E_{0} r$ requires only CVC for $\vec{J}_{V}$, but is an approximation for $\rho_{A}$ made exact by introducing an unknown dimensionless nuclear-structure parameter $\lambda$ that differs from case to case.

[18] T. R. England and B. F. Rider: "Evaluation and Compilation of Fission Product Yields," LA-UR-94-3106 (1994).

[19] Off-equilibrium corrections are needed for fission fragments that decay with half-lives long compared to the timescales involved in the experiments in [3] 5 .

[20] A. A. Borovoi, V. I. Kopeikin, L. A. Mikaelyan, and S. V. Tolokonnikov, Sov. Jour. Nucl. Phys. 36, 232 (1982).

[21] V. I. Kopeikin, Phys. Atomic Nuclei 75143 2012. The mid-cycle combination of burning actinides is $56 \%{ }^{235} \mathrm{U}$, $31 \%{ }^{239} \mathrm{Pu}, 7 \%{ }^{238} \mathrm{U}$ and $6 \%{ }^{214} \mathrm{Pu}$.

[22] P. Vogel, G. K. Schenter, F. M. Mann, and R. E. Schenter, Phys. Rev. C 24, 1543 (1981).

[23] "Schreckenbach-Vogel" means that we used the spectra for ${ }^{235} \mathrm{U},{ }^{239} \mathrm{Pu}$, and ${ }^{241} \mathrm{Pu}$ from [4] and ${ }^{238} \mathrm{U}$ from [22]. 